\title{
Liver function in Edinburgh haemophiliacs: a five-year follow-up
}

\author{
ML STIRLING, GJ BECKETT, AND IW PERCY-ROBB \\ From the Department of Haematology and the University Department of Clinical Chemistry, Royal \\ Infirmary of Edinburgh, UK
}

SUMMARY Liver function was assessed in 38 Edinburgh haemophiliacs. Results before the introduction of NHS intermediate factor VIII concentrate from 1974 onwards were compared with values in 1979. Measurements of serum bile salts in 16 patients as well as conventional liver function tests gave useful evidence of deranged liver function. Deterioration over the five-year follow-up period was seen only in patients on home treatment using large amounts of factor VIII concentrate, and there was no association between cryoprecipitate usage and derangement of liver function.

With the advent of more intensive, effective treatment for haemophilia concern now focuses on the possibly harmful long-term sequelae of transfusion of pooled human plasma products. Cryoprecipitate was introduced into the Edinburgh Haemophilia Centre in 1970 and still provides most of the factor VIII used there. It requires to be stored below $-20^{\circ} \mathrm{C}$, however, and before use is thawed and pooled in batches of six donations upwards for infusion. It is thus inconvenient for use by haemophiliacs on home treatment. In 1974 we began to use the fractionated freeze-dried intermediate factor VIII concentrate produced by the Scottish National Blood Transfusion Service (SNBTS) at its Edinburgh Protein Fractionation Centre. This has a long shelf life and is conveniently stored in small glass bottles at $4^{\circ} \mathrm{C}$ to be made up with sterile water before infusion. It is convenient for use in home treatment, requires a small fluid and total protein load to the patient compared with cryoprecipitate, and is assayed in batches before use so that an exact dose can be calculated. It also tends to produce fewer allergic reactions than cryoprecipitate. The SNBTS product is made from plasma of voluntary local donors and the plasma is pooled in batches of about 600 donations before fractionation. All donors are screened for hepatitis B antigen (HBsAg) carriage by a sensitive radioimmunoassay technique. ${ }^{1}$ A relatively low carriage rate would be expected in this group of voluntary donors. $^{2}$

Even with such screening of blood products from all sources evidence of continued exposure of the haemophilia population in other centres to hepatitis

Accepted for publication 28 May 1980
B virus is strong. ${ }^{3-5}$ 'Non-A, non-B' viral hepatitis is also increasingly reported in these patients ${ }^{6}$ and there is a considerable amount of pathological evidence of chronic liver disease in haemophiliacs, including chronic persistent hepatitis, chronic aggressive hepatitis, and micronodular cirrhosis. ${ }^{3} 78$ We report the findings of liver function tests in patients attending the Edinburgh Haemophilia Centre, including data preceding the introduction of factor VIII concentrate in 1974. This group of patients is unique in being exposed to concentrate only from the local blood transfusion service and not, as in other centres, commercial concentrate. Abnormalities of liver function in these patients and, in particular, evidence of deterioration since the introduction of pooled concentrate are therefore of considerable interest.

\section{Patients and methods}

Data were collected from 38 patients, of whom seven had been treated almost exclusively with concentrate at home. The remaining 31 patients had received predominantly cryoprecipitate on demand at the haemophilia centre, and had had varying exposure to concentrate for periods of intensive inpatient treatment, elective surgical procedures, and occasional holiday use. Data were available before first exposure to concentrate between 1974 and 1976, and the results were compared with the corresponding 1979 values. In those patients in whom blood had been examined frequently the results nearest the time concentrate was introduced were selected. The 1979 values were obtained at a time of presentation to the haemophilia centre for treatment or when 
stocks for home treatment were issued. Infrequent attenders reported for blood checks on request. Serum bilirubin, alanine aminotransferase (ALT), alkaline phosphatase, and hepatitis B surface antigen and antibody were measured in all patients, and in 16, including the seven being treated at home, hepatic anion transport was also assessed by measuring fasting and post-prandial bile salts. This latter measurement is a sensitive method of detecting early liver damage. ${ }^{910}$

Plasma concentrations of bilirubin, ALT, and alkaline phosphatase were measured by a Sequential Multiple Analysis with Computer (SMAC) system (Technicon Instruments Corporation, Basingstoke, UK). Bile salts (conjugated cholic acid and conjugated chenodeoxycholic acid) were measured by a radioimmunoassay method.1112 Fasting concentrations and half-hourly samples for two hours after a standard fatty meal $(150-\mathrm{ml}$ fruit yoghurt plus $150-\mathrm{ml}$ double cream) were analysed. Bile salt concentrations above the reference range indicated abnormal hepatic anion transport, which is an index of the presence of liver disease.

The patients were subdivided into the following three groups: (1) home treatment group with exposure to many $(>20)$ different batches of concentrate (seven patients); (2) mainly cryoprecipitate but also from 10-20 batches of concentrate (eight patients); (3) mainly cryoprecipitate and $<10$ batches of concentrate ( 23 patients).

The number of different batches of concentrate was used for analysis rather than the total number of units, since this was thought to reflect more accurately exposure to potential hepatotoxic viruses or other substances in the concentrate. One patient received no treatment at all in the three-year follow-up period, and one received cryoprecipitate only and no concentrate. Both were included in group 3. In groups 2 and 3 the 1979 results were also compared with their mean annual cryoprecipitate usage over the three-year period 1976-8, for which accurate records were available.

\section{Results}

No patient had a raised plasma total bilirubin concentration, and alkaline phosphatase activities were raised only in those 15 patients who were in an adolescent growth phase. None of the patients had symptoms or objective clinical evidence of liver disease.

\section{ALT ACTIVITIES}

In group 1 , two patients had abnormal concentrations of ALT in 1974-6, whereas six out of seven were abnormal in 1979 (Figure). One of the patients who was abnormal in the first measurement became more abnormal by 1979 . The other was only marginally abnormal on both occasions. In group 2 four out of eight patients were abnormal in 1974-6, though one only slightly so. Three remained abnormal in 1979, while one fell to within the normal range. One patient who was normal in 1974-6 subsequently became abnormal. Group 3 showed a wide variation in results. Seven out of 23 patients were abnormal in 1974-6, and five out of the seven became normal and two fell but were still outwith the normal range. A further five patients who were normal in 1974-6 were abnormal by 1979. Thus evidence of a deterioration in ALT concentrations was apparent only in group 1 , with groups 2 and 3 showing no overall significant change.

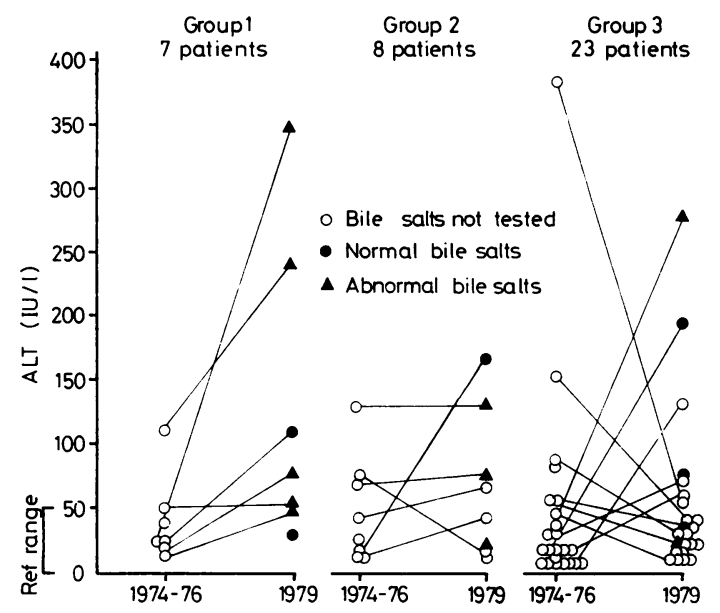

Alanine aminotransferase $(A L T)$ activities in 38 haemophiliacs.

In the patients on home treatment, in whom regular checks on liver function were carried out, wide fluctuations in ALT activities were noted. In general, these changes did not appear to be related in time to the injection of blood products, but in one patient a transient rise in ALT activity was seen two weeks after exposure to a single batch of concentrate for a dental extraction, and in a further patient a prolonged rise in ALT followed the use of multiple infusions for surgery. In groups 2 and 3 the ALT concentrations were compared with the average amount of cryoprecipitate used annually by each patient over a three-year period, 1976-8, and no correlation was found.

BILE SALT STUDIES

Plasma bile salt concentrations, both conjugated 
cholate and chenodeoxycholate, in fasting and postprandial specimens were measured in 16 patients (Table, Figure). The seven home treatment patients were all included in this part of the study. Five of them had at least one abnormal bile salt result, and four of these five had abnormal ALT concentrations. One patient with normal ALT activity showed evidence of liver damage on bile salt studies alone. The other nine patients were from groups 2 and 3 . Four were abnormal, four within normal limits, and one (case 15) showed raised fasting bile salt concentrations without a post-prandial rise, suggesting gall bladder dysfunction. Two patients with abnormal bile salt concentrations in this group had normal ALT concentrations and, conversely, only one patient with abnormal ALT concentrations showed a normal bile salt profile. Thus ALT and bile salt measurements generally correlated in identifying patients with biochemical liver disease.

HEPATITIS SURFACE ANTIGEN AND ANTIBODY One of the patients in group 1 was a chronic carrier of HBsAg throughout the period of study. His ALT concentrations were normal initially but abnormal in 1979, as were bile salt studies. In 1974-6 two other patients in this group had detectable antibody to HBsAg before embarking on home treatment, and by 1979 five were antibody-positive. Only one patient was negative for antibody on both occasions. One patient in group 2 was positive for antibody in 1974-6 and the remaining seven negative. By 1979 five were positive and three were negative. Five of the 25 patients in group 3 were positive for antibody in 1974-6. One of them became negative by 1979 and a further 11 converted to positive antibody status- that is, 15 out of 23 in all. Thus 18 out of the total 38 patients showed evidence of exposure to hepatitis $B$ after the introduction of factor VIII concentrate. One patient developed clinical serum hepatitis after a single batch of concentrate for dental extractions, with HBs antigenaemia persisting for several weeks. He later became antibody-positive and his ALT and bilirubin concentrations returned to normal. No other blood products had been given and the batch of concentrate was subsequently withdrawn.

\section{Discussion}

There is serological evidence of continued exposure to hepatitis B among the haemophiliacs in this study despite careful screening of donors. Almost half the patients developed antibody to HBsAg after the introduction of factor VIII concentrate and one had clinical hepatitis B.

There is evidence of abnormal liver function in many of the patients, but only in those in group 1, who received large amounts of concentrate, is there evidence of deterioration since 1974-6. Groups 2 and 3 , where more cryoprecipitate and less concentrate was used, show no evidence of worsening over the study period and there is no direct correlation between abnormal ALT concentrations and amounts of cryoprecipitate used. Measurement of serum bile salts has been a useful index of liver disease in these patients, in whom the plasma total bilirubin concentrations were all normal. Close correlation with ALT values is shown, but in two patients without other abnormal parameters bile salt studies provide useful evidence of occult liver damage. They also support the assumption that rises

Fasting and two-hour post-prandial serum bile salt concentrations (abnormal results underlined) in 16 patients with haemophilia

\begin{tabular}{|c|c|c|c|c|c|c|}
\hline \multirow[t]{2}{*}{ Patient group No. } & \multirow[t]{2}{*}{ Case No. } & \multicolumn{2}{|l|}{ Fasting ( $\mu \mathrm{mol} / \mathrm{l})$} & \multicolumn{2}{|l|}{ Post-prandial ( $\mu \mathrm{mol} / \mathrm{l})$} & \multirow[t]{2}{*}{ Hepatic anion trasport } \\
\hline & & Chenodeoxycholate & Cholate & Chenodeoxycholate & Cholate & \\
\hline $\begin{array}{l}1 \\
1 \\
1 \\
1 \\
1 \\
1 \\
1 \\
2 \\
2 \\
2 \\
2 \\
3 \\
3 \\
3 \\
3 \\
3\end{array}$ & $\begin{array}{r}1 \\
2 \\
3 \\
4 \\
5 \\
6 \\
7 \\
8 \\
9 \\
10 \\
11 \\
12 \\
13 \\
14 \\
15 \\
16\end{array}$ & $\begin{array}{l}\frac{8.0}{1.9} \\
2.6 \\
1.5 \\
0.7 \\
\frac{5.7}{1 \cdot 1} \\
0.2 \\
1.7 \\
\frac{5.0}{2.2} \\
0.9 \\
1.2 \\
0.9 \\
\frac{4.1}{2.5}\end{array}$ & $\begin{array}{l}\frac{6.5}{0.9} \\
2.4 \\
1.4 \\
1.9 \\
\frac{6.3}{1 \cdot 1} \\
3 \cdot 2 \\
1.9 \\
4 \cdot 3 \\
3 \cdot 0 \\
2.5 \\
2.4 \\
1 \cdot 1 \\
5.0 \\
4.9 \\
\end{array}$ & $\begin{array}{r}\frac{14.8}{13.4} \\
6.9 \\
7 \cdot 2 \\
17.0 \\
10 \cdot 1 \\
20.6 \\
8.5 \\
7.9 \\
16.7 \\
23.7 \\
10.6 \\
8.6 \\
4.0 \\
4.1 \\
5.6\end{array}$ & $\begin{array}{l}\frac{12 \cdot 2}{13 \cdot 3} \\
\frac{8 \cdot 4}{5 \cdot 3} \\
\frac{18 \cdot 7}{7 \cdot 2} \\
\frac{9 \cdot 4}{11 \cdot 3} \\
5 \cdot 5 \\
\frac{19 \cdot 4}{22.9} \\
8 \cdot 6 \\
7 \cdot 1 \\
3.0 \\
5.0 \\
5.0\end{array}$ & $\begin{array}{l}\text { Abnormal } \\
\text { Abnormal } \\
\text { Normal } \\
\text { Normal } \\
\text { Abnormal } \\
\text { Abnormal } \\
\text { Abnormal } \\
\text { Abnormal } \\
\text { Normal } \\
\text { Abnormal } \\
\text { Abnormal } \\
\text { Normal } \\
\text { Normal } \\
\text { Normal } \\
\text { Abnormal } \\
\text { Abnormal }\end{array}$ \\
\hline Reference range & & $0 \cdot 2-2 \cdot 9$ & $0 \cdot 2-3 \cdot 2$ & $1 \cdot 0-13 \cdot 5$ & $1 \cdot 1-8 \cdot 3$ & \\
\hline
\end{tabular}


in ALT activities in these patients are hepatic in origin and not simply due to trauma, anaesthesia, or other stresses.

Transient rises in ALT do occur, and values in any one patient may fluctuate considerably. The nature of these fluctuations and their clinical significance remains uncertain. Possible causes include repeated infection with as yet unidentified non-A, non-B hepatitis viruses and hepatic damage after the formation of antigen-antibody complexes in patients with immunity exposed to repeated insult by viral and other antigens.

The nature and permanence of liver damage in these patients remains obscure. As none was in any way symptomatic or showed clinical evidence of liver disease we did not feel justified in submitting any of them to liver biopsy. This remains a possibility for the future. Meanwhile there does not appear to be sufficient evidence of any serious deterioration in liver function from NHS concentrate to limit its current use for patients on home treatment, for whom the convenience of the product is all important. It would seem reasonable, however, that patients in hospital should whenever possible receive nonpooled cryoprecipitate instead. Comparison with follow-up results of liver function in haemophiliacs exposed to commercial factor VIII concentrate would be useful.

We thank Dr SH Davies for permission to study these patients.

\section{References}

${ }^{1}$ Hopkins R, Ross S, Jordan T, Watt AD. Improved economics of HBsAg screening with commercial radioimmunoassay agents. J Clin Pathol 1980;33:19-23.
2 Hopkins R, Robertson AE, Ravie A, McClelland DBL. Blood donors with history of jaundice. Lancet 1980; ; :596.

${ }^{3}$ Mannucci PM, Ronch G, Rota L, Colombo M. A clinicopathological study of liver disease in haemophiliacs. J Clin Pathol 1978;31:779-83.

4 Peterson MR, Barker LF, Schade DS. Detection of antibody to hepatitis associated antigen in hemophilia patients and in voluntary blood donors. Vox Sang 1973;24:66.

${ }^{5}$ Hasiba UW, Spero JA, Lewis JH. Chronic liver dysfunction in multitransfused hemophiliacs. Transfusion 1977;17: 490-4.

${ }^{6}$ Craske J, Spooner RJD, Vandervelde EM. Evidence for existence of at least two types of factor VIII associated non B transfusion hepatitis. Lancet 1978;ii:1051-2.

7 Preston FE, Underwood JCE, Mitchell VE, Triger DR, Bardhan G, Stewart RM, Blackburn EK. Percutaneous liver biopsy and chronic liver disease in haemophiliacs. Lancet 1978; ii:592-4.

${ }^{8}$ Hilgartner MW, Giardina P. Liver dysfunction in patients with hemophilia A, B and von Willebrand's disease. Transfusion 1977;17:495-9.

${ }^{9}$ Kaplowitz W, Kok MS, Javitt NB. Post prandial serum bile acids for the detection of hepatobiliary disease. JAMA 1973;225:292-3.

${ }^{10}$ Korman MG, Hofmann AF, Summerskill WHJ. Assessment of activity in chronic active liver disease; serum bile acids compared with conventional tests and histology. New Eng J Med 1974;290:1399-402.

${ }^{11}$ Beckett GJ, Hunter WM, Percy-Robb IW. Investigation into the choice of immunogen, ligand, antiserum and assay conditions for the radioimmunoassay of conjugated bile acids. Clin Chem Acta 1978;88:257-66.

12 Beckett GJ, Corrie JET, Percy-Robb IW. The preparation of ${ }^{125} \mathrm{I}$ labelled bile acid ligands for use in the radioimmunoassay of bile acids. Clin Chem Acta 1979;93: 145-50.

Requests for reprints to: Dr ML Stirling, Department of Haematology, Royal Infirmary of Edinburgh, Lauriston Place, Edinburgh EH3 9YW. 